\title{
A Hybrid Morphological Active Contour FOR NATURAL IMAGES
}

\author{
Victoria L. Fox ${ }^{1}$, Mariofana Milanova ${ }^{2}$, Salim Al-Ali $^{3}$ \\ ${ }^{1}$ Department of Applied Science, University of Arkansas at Little Rock, USA \\ vlfoxeualr.edu \\ ${ }^{2}$ Department of Computer Science, University of Arkansas at Little Rock, USA \\ mgmilanova@ualr.edu \\ ${ }^{3}$ Department of Computer Science, University of Arkansas at Little Rock, USA \\ sgsaeedeualr.edu
}

\begin{abstract}
Morphological active contours for image segmentation have become popular due to their low computational complexity coupled with their accurate approximation of the partial differential equations involved in the energy minimization of the segmentation process. In this paper, a morphological active contour which mimics the energy minimization of the popular Chan-Vese Active Contour without Edges is coupled with a morphological edge-driven segmentation term to accurately segment natural images. By using morphological approximations of the energy minimization steps, the algorithm has a low computational complexity. Additionally, the coupling of the edge-based and region-based segmentation techniques allows the proposed method to be robust and accurate. We will demonstrate the accuracy and robustness of the algorithm using images from the Weizmann Segmentation Evaluation Database and report on the segmentation results using the Sorensen-Dice similarity coefficient.
\end{abstract}

\section{KEYWORDS}

Natural Images, Segmentation,Hybrid Contour, Morphology, Active Contours, Object Extraction

\section{INTRODUCTION}

One goal of natural image segmentation is to accurately mimic the object recognition and scene analysis of human visual perception. While organic perceptual systems efficiently segment images into homogenous regions [1], obtaining similar results is a challenging problem for computational image segmentation. The difficulty in computational processing of natural images can be attributed to inherent statistical complexities of the image and a lack of homogeneity or saliency of local features at the same spatial or quantization scale [2]. As a result, segmentation of natural images remains a challenging task in image processing and computer vision.In recent literature, methods for segmentation of natural images include active contours [3-5], clustering methods [69],lossy data compression [10,11] and graph cuts [12].

\subsection{Active Contour Models}

In this work, we are interested in unsupervised, active contour models for segmentation. The method of image segmentation by active contours can be divided into three approaches: edge based, region based, and hybrid. Edge based active contours represented by [4]and the references therein use an energy driven by attraction to the edges of regions of interest in the image. For DOI : $10.5121 /$ ijcsea.2013.3401 
accuracy, the object of interest should have obvious boundaries usually represented by a dramatic change in the gradient values of the image. In the case of natural images, edges of the object of interest are not always clearly delineated and edge based methods often require more complex algorithms to achieve some degree of accuracy in the segmentation. Additionally, edge-based algorithms must be carefully formulated so that image gradient changes due to lighting, background objects, and noise do not cause the contour to inaccurately segment the image. This requirement also adds to the complexity of edge-based segmentation models.

Region based segmentation models [5, 13-18] use image statistics to partition the image into regions. Ideally, the object of interest will have different statistics in comparison to surrounding areas and can then be quickly segmented. As is often the case, however, natural images have a vast number of visual and textural patterns and are inherently noisy. Traditional methods work to resolve the problem by incorporating local statistics in the contour evolution [16-18] which has led to a new set of issues regarding the accuracy of the segmentation. While accuracy is greatly improved when statistical models include local information, these methods often still fail to fit the contour to the object boundaries due to a lack of edge information and they are sensitive to the size of the window used to calculate local statistics [15]. Other region based segmentation algorithms frequently incorporate some type of texture analysis, e.g. [13] and its references, into a pre-processing step prior to region segmentation. While the texture analysis greatly enhances the accuracy of the model, it also contributes to the complexity and computational cost.

More recently, hybrid models which incorporate both edge and region information in their segmentation algorithms have been introduced [19-23]. In hybrid models, a strong edge term attracts the curve toward objects of interest while a region-based term propagates the curve when the edge information is absent. While hybrid methods have found success in medical segmentation applications [19, 21-22], there is a lack of literature involving the accuracy of hybrid methods with respect to natural images. To the authors' knowledge, while [23] mentions natural image segmentation and provides a simple example, a robust study of the effectiveness of a hybrid strategy for natural image segmentation has not been conducted. This may be due to the shortcomings of both methods in conjunction with the intensity inhomogeneity and statistical complexity of natural images does not allow an efficient, robust algorithm when attempted with traditional hybrid methods. If one was to use a hybrid active contour method for natural image segmentation, it could be construed that it would have to be a method which uses a simpler computational scheme than the statistical and energy minimization schemes of regional and edge based methods while enhancing the accuracy of the segmentation.

\subsection{Morphological Active Contours}

One method of morphological segmentation is achieved through repeated median filtering with an adaptive structuring element which has been shown to be a self snake [24]. While mathematically sound, the process in itself has a high computational cost since it is achieved by filtering pixel-by-pixel. A less computationally expensive method of segmentation through morphological structuring elements is the proven practice of using morphology to approximate the partial differential equations used in traditional active contour models. The morphological evolution solves the traditional partial differential equations but avoids many issues associated with numerical algorithms.

In edge based active contour methods, the contour is composed of three components: a balloon force, a smoothing force, and an edge attraction force. A region based active contour typically has three terms as well: a balloon force, a statistical region force, and a smoothing force. For the morphological approximation of the forces, it is well known that erosion or dilation will approximate the balloon force of the formulation. The edge attractant force and region forces can 
be approximated discretelyby comparison of image gradients and region statistics, respectively. However, until recently, there was not a morphological equivalent to the smoothing term and pseudo-morphological active contours still used partial differential equations to smooth the contour.

In partial differential equation formulations of active contours, the smoothing force regularly takes the form of mean curvature motion and acts as a regularization term. The underlying principal of mean curvature motion is the evolution of a simple closed curve whose points move in the direction of the normal with specified velocity.

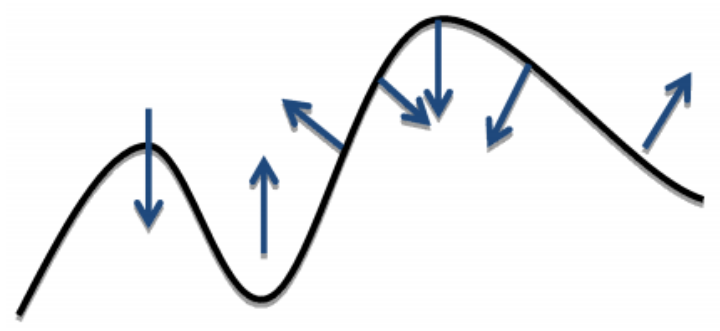

Figure 1: Mean Curvature Motion

Catte, Dibos, and Koepfler [25] advanced the topic of creating a discrete smoothing force by proving the two-dimensional mean curvature term can be replaced by the mean of two morphological operators for a single iteration of the method. To do this, we let $\mathcal{B}$ represent line segments of set length then define the morphological continuous line operators as

$$
\begin{aligned}
& \left(\mathcal{A}_{h} u\right)(\boldsymbol{x})=\sup _{B \in \mathcal{B} \boldsymbol{y} \in \boldsymbol{x}-h B} u(\boldsymbol{y}), \\
& \left(\mathcal{J}_{h} u\right)(\boldsymbol{x})=\inf _{B \in \mathcal{B}} \boldsymbol{\operatorname { s u p }} \in \boldsymbol{x}+h B \\
& B(\boldsymbol{y}) .
\end{aligned}
$$

Using these operators, we thendefine the mean operator as

$$
\left(\mathcal{F}_{h} u\right)(\boldsymbol{x})=\frac{\left(\mathcal{A}_{h} u\right)(\boldsymbol{x})+\left(\mathcal{J}_{h} u\right)(\boldsymbol{x})}{2}
$$

in which the scheme in [25] relates the mean operator with the mean curvature motion by

$$
\left(\mathcal{F}_{h} u\right)(\boldsymbol{x})=u(\boldsymbol{x})+.25 h^{2}|\nabla u| \operatorname{div}\left(\frac{\nabla u}{|\nabla u|}\right)(\boldsymbol{x})+O\left(h^{3}\right)
$$

Using a small $h$ and subtracting $u(x)$ from each side of (4) results in the infinitesimal generator of the $\mathcal{F}_{h}$ operator:

$$
\lim _{h \rightarrow 0^{+}} h^{-1}\left[\left(\mathcal{F}_{\sqrt{4 h}} u\right)(\boldsymbol{x})-u(\boldsymbol{x})\right]=|\nabla u| \operatorname{div}\left(\frac{\nabla u}{|\nabla u|}\right)(\boldsymbol{x}) .
$$

From (5) we can solve the mean curvature motion by means of the $\mathcal{F}_{h}$ operator. However, since the $\mathcal{F}_{h}$ operator generates new level set values after a single iteration, it ceases to be morphological. In [26] and [27], Alarez et al. modify the Catte, Dibos, and Koepfler scheme with the use of operator composition which states that given any two operators $\mathcal{P}_{h}^{1}$ and $\mathcal{P}_{h}^{2}$, we have, for a small $h$,

$$
\mathcal{P}_{h / 2}^{2} \circ \mathcal{P}_{h / 2}^{1} u \approx \frac{\mathcal{P}_{h}^{2} u+\mathcal{P}_{h}^{1} u}{2} .
$$


From this, Alarez et al. show that the non-morphological operator $\mathcal{F}_{\sqrt{4 h}}$ can be approximated by the morphological operator $\mathcal{A}_{\sqrt{h}} \circ \mathcal{J}_{\sqrt{h}}$ with a base of $\mathcal{B}^{2}$. With this morphological operator in place, it is now possible to develop a discrete morphological active contour for edge based and region based active contours. Results of using discrete, morphological active contours in [26] and [27] show a much lower computational cost and a subsequent increase in computational speed.

Unfortunately, approaching segmentation of natural images by a solely edge-based or regionbased morphological active contour does not result in any higher accuracyin segmentation results. For example, the natural images used in [27]- of which there are two - still show the segmentation flaws of their partial differential equation counterparts. Despite this, the contributions of Alarez and his co-authors are extremely significant due to the mathematical validation of a morphological mean curvature approximation and the formulation of discrete, morphological algorithms for both edge based and region based methods.

\subsection{Contribution}

In our approach, we contend that a more accurate morphological active contour for natural images can be obtained if we combine positives of the edge-based methods with region statistics. The proposed method uses the morphological equivalent of an edge-driven balloon force coupled with region statistics and morphological mean curvature motion to segment natural images. The use of morphological operators results in an efficient implementation of the algorithm while the hybrid contour accurately segments natural images when compared to ground-truth. Using the Sorenson-Dice similarity coefficient to evaluate accuracy, we found our segmentation of the images provided by the Weizmann Segmentation Evaluation Database averaged a 0.9302 similarity ratio. In short, the proposed method is simple, efficient, and robust for the segmentation of natural images. Additionally, the use of morphological operators and edge-based balloon forces resulted in accurate segmentation of multiphase images in the dataset.

\section{Hybrid Morphological ACTIVE CONTOUR}

The idea to combine edge and region information in a segmentation algorithm is not new. Usually, the combination results in increased computational complexity to mitigate the shortcomings of either edge based or region based segmentation or both[15]. The advantage of a hybrid morphological contour is both the edge based and region based methods have low complexity and circumventing a method's shortcoming does not significantly add to the computational cost. For example, in order to control illumination issues or noise, one need only alter the structuring element in either the balloon force or smoothing term. The hybrid method uses a zero level set of a binary piecewise constant function $u: \mathbb{R}^{2} \rightarrow\{0,1\}$. The morphological operators act on $u$ and implicitly evolve the curve.

\subsection{Balloon Force}

The edge-based portion of the method is represented by an edge-driven balloon force. In edge based methods, the contourflow is often represented with

$$
\frac{\partial u}{\partial t}=g(I)|\nabla u| v+\nabla g(I) \nabla u+g(I)|\nabla u| \operatorname{div}\left(\frac{\nabla u}{|\nabla u|}\right)
$$

where $g(I)|\nabla u| v$ is the balloon force, $\nabla g(I) \nabla u$ is the edge attraction force, and $g(I)|\nabla u| \operatorname{div}\left(\frac{\nabla u}{|\nabla u|}\right)$ is mean curvature motion. $g(I)$ represents an edge image obtained from an edge detector, $u$ denotes the contour, and vis an inflation (or deflation) constant. Focusing on the 
balloon force, $g(I)$ could be obtained from any edge detector appropriate for the image. Traditionally, one would use an edge detector which is low in the edges of the image such as

$$
g(I)=\frac{1}{\sqrt{1+\alpha\left|\nabla G_{\sigma^{*}}\right|}} .
$$

In the proposed method, morphological operations of dilation and erosion are used to approximate the balloon force. The dilation of a function is defined as $\left(\mathcal{D}_{h} u\right)(\boldsymbol{x})=$ $\sup _{\boldsymbol{y} \in h B} u(\boldsymbol{x}-\boldsymbol{y})$ while erosion is defined by $\left(\mathcal{E}_{h} u\right)(\boldsymbol{x})=\inf _{\boldsymbol{y} \in h B} u(\boldsymbol{x}-\boldsymbol{y})$. The radius of the operator is denoted by $h$ and $B$ is a disk structuring element of radius one.The function $u_{d}: \mathbb{R}^{+} \times$ $\mathbb{R}^{2} \rightarrow \mathbb{R}$ where $u_{d}(t, \boldsymbol{x})=\mathcal{D}_{t} u_{o}(\boldsymbol{x})$ is the solution to

$$
\frac{\partial u_{d}}{\partial t}=\left|\nabla u_{d}\right|
$$

for the initial condition $u_{d}(0, \boldsymbol{x})=u_{o}(\boldsymbol{x})$ [28]. As a result, $\mathcal{D}_{h}$ is the infinitesimal generator of (9). Using a comparable rational, we have the function $u_{d}: \mathbb{R}^{+} \times \mathbb{R}^{2} \rightarrow \mathbb{R}$ where $u_{e}(t, \boldsymbol{x})=$ $\mathcal{E}_{t} u_{o}(\boldsymbol{x})$ is the solution to

$$
\frac{\partial u_{e}}{\partial t}=-\left|\nabla u_{e}\right|
$$

withinitial condition $u_{e}(0, \boldsymbol{x})=u_{o}(\boldsymbol{x})$. Using the morphological operators $\mathcal{D}_{h}$ and $\mathcal{E}_{h}$, we can now solve level set evolution PDEs like equations 9 and 10. In the balloon force term, $g(I)$ manages the balloon force in individual sections of the curve. The smaller $g(I)$ becomes, the closer the curve is to the edge. With the use of a threshold, factor $g(I)$ can be discretized into the morphological formulation. The product $|\nabla u| v$ leads to equations the PDES in equations 9 and 10. If $v$ is positive, the PDE becomes the dilation PDE. Likewise, if $v$ is negative, then the erosion PDE is used. Therefore, the solution to the balloon force PDE applied over the contour $u: \mathbb{R}^{2} \rightarrow\{0,1\}$ can determined with the following morphological method using discrete dilation, $\mathcal{D}_{d}$ and discrete erosion $\mathcal{E}_{d}$ :

$$
u_{\text {balloon }}^{n}\left(\boldsymbol{x}_{\boldsymbol{i}}\right)=\left\{\begin{array}{c}
\left(\mathcal{D}_{d} u\right)\left(\boldsymbol{x}_{i}\right) \text { if } g(I)\left(\boldsymbol{x}_{i}\right)>\text { threshold and } v>0 \\
\left(\mathcal{E}_{d} u\right)\left(\boldsymbol{x}_{i}\right) \text { if } g(I)\left(\boldsymbol{x}_{i}\right)>\text { threshold and } v<0 \\
u_{\text {balloon }}^{n-1} \text { otherwise }
\end{array}\right.
$$

\subsection{Region Statistics}

In the proposed hybrid method, the coupling of the strong edge term and region statistics creates a symbiotic relationship. When the edge term is low, the curve is attracted toward the region of interest. However, when the curve is far away from an edge, the region statistics take control of the curve evolution and the contour resists becoming a stationary model. While there are several different statistics that can be of use in region based segmentation, the use of intensity statistics provided accurate and efficient guidance for our segmentation experiments.

A common region based model, the Chan-Vese Active Contour without Edges [29], gives the following functional of a curve $\mathcal{C}$ :

$$
\begin{aligned}
F\left(c_{1}, c_{2}, \mathcal{C}\right)= & \mu(\text { Length of } \mathcal{C})+p(\text { Area in } \mathcal{C})+\lambda_{1} \int_{\text {in }}\left|u_{0}-c_{1}\right|^{2} d x \\
& +\lambda_{1} \int_{\text {out }}\left|u_{0}-c_{2}\right|^{2} d x
\end{aligned}
$$


where $c_{1}$ and $c_{2}$ are the average intensity levels inside and outside the contour. The constant $\mu$ penalizes the length of the contour while $p$ limits the area inside the contour. Parameters $\lambda_{1}$ and $\lambda_{2}$ weight the importance of the regions inside and outside the curve, respectively. To ease computation of the minimization of the functional, it is noted that

$$
A\{u \geq 0\}=\int_{\Omega} H(u) d x
$$

in which $H(u)$ is a signed, step function known as the Heaviside function and $\Omega$ is the image domain. The impact of equation 12 for the intensity terms of the Chan-Vese function is demonstrated in the following equations:

$$
\begin{array}{r}
\lambda_{1} \int_{\text {in }}\left|u_{0}-c_{1}\right|^{2} d x=\lambda_{1} \int_{u>0}\left|u_{0}-c_{1}\right|^{2} d x=\lambda_{1} \int_{\Omega}\left|u_{0}-c_{1}\right|^{2} H(u) d x \\
\lambda_{2} \int_{\text {out }}\left|u_{0}-c_{2}\right|^{2} d x=\lambda_{1} \int_{u<0}\left|u_{0}-c_{2}\right|^{2} d x=\lambda_{1} \int_{\Omega}\left|u_{0}-c_{2}\right|^{2}(1-H(u)) d x
\end{array}
$$

In short, the intensity terms can now be computed using the image domain rather than the region domain.

In [27], it is shown that the Active Contours without Edges functional can be minimized with a morphological active contour. Once again, the functional is comprised of three energies: balloon force, region force, and a smoothing force. The balloon force is represented by $p$ (Area in $\mathcal{C})$, the region force is denoted by $\mu($ Length of $\mathcal{C})$. The morphological equivalence of the balloon force is given by

$$
u_{\text {balloon }}^{n}\left(\boldsymbol{x}_{\boldsymbol{i}}\right)=\left\{\begin{array}{c}
\left(\mathcal{D}_{d} u\right)\left(\boldsymbol{x}_{i}\right) \quad p>0 \\
\left(\mathcal{E}_{d} u\right)\left(\boldsymbol{x}_{i}\right) p<0 \\
u_{\text {balloon }}^{n-1} \text { otherwise } .
\end{array}\right.
$$

The authors of [27] did not use the Heaviside equation in the calculation of the region statistics. Using the image intensity terms from the original Chan-Vese equation, the region term can be discretely modelled by

$$
u_{\text {region }}^{n}=\left\{\begin{array}{c}
1 \quad \text { if }\left|\nabla u_{\text {balloon }}^{n}\right|\left[\left(\lambda_{1}\left(I-c_{1}\right)^{2}-\lambda_{2}\left(I-c_{2}\right)^{2}\right]\left(\boldsymbol{x}_{\boldsymbol{i}}\right)<0\right. \\
0 \text { if } \mid \nabla u_{\text {balloon }}^{n}\left[\left(\lambda_{1}\left(I-c_{1}\right)^{2}-\lambda_{2}\left(I-c_{2}\right)^{2}\right]\left(\boldsymbol{x}_{\boldsymbol{i}}\right)>0\right. \\
u_{\text {region }}^{n-1} \text { otherwise }
\end{array}\right.
$$

where $c_{1}$ and $c_{2}$ are the mean of the values of intensity inside and outside the contour. However, in terms of computational stability and robustness, the use of equations 13 and 14 in our proposed hybrid method greatly increase the accuracy of the method. As a result, our region statistics term uses the following to compute $c_{1}$ and $c_{2}$ :

$$
\begin{aligned}
& c_{1}=\frac{\int_{\Omega^{*} I H(u) d x}}{\int_{\text {inside } e^{d x}}} \\
& c_{2}=\frac{\int_{\Omega^{I *} I(1-H(u)) d x}}{\int_{\text {inside } e^{d x}}}
\end{aligned}
$$

\subsection{Mean Curvature Motion}

The smoothing term in the hybrid morphological active contour takes the form of the morphological equivalent of mean curvature motion. Recall from the geodesic active contour 
method expressed in equation 7 , the mean curvature motion is given by $g(I)|\nabla u| \operatorname{div}\left(\frac{\nabla u}{|\nabla u|}\right)$. Using equations 5 and 6 and a simple threshold for $g(I)$, the morphological evolution of the mean curvature motion is

$$
u_{m c m}^{n}=\left\{\begin{array}{cc}
\left(\mathcal{A}_{d} \circ \mathcal{J}_{d} u_{\text {region }}^{n}\right)\left(\boldsymbol{x}_{\boldsymbol{i}}\right) & \text { if } g(I)(\boldsymbol{x})>\text { threshold } \\
u_{\text {region }}^{n} & \text { otherwise }
\end{array}\right.
$$

Both $\mathcal{A}_{d}$ and $\mathcal{J}_{d}$ have a discrete version of $\mathcal{B}$, denoted $\mathcal{L}$, which is a collection of four discretized segments centered at the origin of a set length. A natural explanation of the morphological mean curvature motion operator involves applying it to a binary image. In a binary image, $\mathcal{A}_{d}$ works only on white pixels and $\mathcal{J}_{d}$ works on black pixels. Suppose $u\left(\boldsymbol{x}_{0}\right)$ is a black pixel. Then $i n f_{\boldsymbol{y} \in \boldsymbol{x}_{0}+\mathcal{L}} u(\boldsymbol{y})$ will be zero for every segment $\operatorname{Lin} \mathcal{L}$. Similarly, $\mathcal{J}_{d}$ does not affect white pixels.

For every white pixel, $\boldsymbol{x}_{w}$, in a binary image, the $\mathcal{A}_{d}$ operator looks for line segments of a set pixel length which cover $\boldsymbol{x}_{w}$. This search is done in an eight-connected component pattern. If no line exists, then the pixel is made black. For black pixels, $\mathcal{J}_{d}$ performs similarly. The composition $\mathcal{A}_{d} \circ \mathcal{J}_{d}$ first eliminates unconnected black pixels with $\mathcal{J}_{d}$ and then repeats the procedure for white pixels with $\mathcal{A}_{d}$. The result is a smoothing of the contour for eachrepetition of the algorithm.

\section{IMPLEMENTATION}

The hybrid morphological active contour is comprised of two edge based terms and one region based term. Using an edge attraction term, region statistics with $c_{1}$ and $c_{2}$ computed with the Heaviside function, and the morphological mean curvature motion, we can formulate the active contour algorithm.

\subsection{Algorithm}

In PDE based active contours, the balloon, image attraction, and smoothing forces are combined through addition of the terms. Our hybrid morphological active contour will combine them by iteratively interchanging their discretized formulations. In every iteration, we will first apply the balloon force with the edge attraction energy (11), then apply the region force (16), and finish with the mean curvature motion (19) over the embedded level set function $u$. Given the contour evolution at iteration, $u^{n}: \mathbb{R}^{2} \rightarrow\{0,1\}$, we define $u^{n+1}$ using the following steps:

Step 1:

$$
u_{\text {balloon }}^{n+1}\left(\boldsymbol{x}_{\boldsymbol{i}}\right)=\left\{\begin{array}{c}
\left(\mathcal{D}_{d} u\right)\left(\boldsymbol{x}_{i}\right) \text { if } g(I)\left(\boldsymbol{x}_{i}\right)>\text { threshold and } v>0 \\
\left(\mathcal{E}_{d} u\right)\left(\boldsymbol{x}_{i}\right) \text { if } g(I)\left(\boldsymbol{x}_{i}\right)>\text { threshold and } v<0 \\
u_{\text {balloon }}^{n} \text { otherwise }
\end{array}\right.
$$

Step 2: $\quad u_{\text {region }}^{n+1}=\left\{\begin{array}{cc}1 & \text { if }\left|\nabla u_{\text {balloon }}^{n+1}\right|\left[\left(\lambda_{1}\left(I-c_{1}\right)^{2}-\lambda_{2}\left(I-c_{2}\right)^{2}\right]\left(\boldsymbol{x}_{\boldsymbol{i}}\right)<0\right. \\ 0 & \text { if }\left|\nabla u_{\text {balloon }}^{n+1}\right|\left[\left(\lambda_{1}\left(I-c_{1}\right)^{2}-\lambda_{2}\left(I-c_{2}\right)^{2}\right]\left(\boldsymbol{x}_{\boldsymbol{i}}\right)>0\right. \\ u_{\text {ballon }}^{n+1}\left(\boldsymbol{x}_{i}\right) \text { otherwise }\end{array}\right.$

$$
\text { where } \quad c_{1}=\frac{\int_{\Omega} I * H(u) d x}{\int_{\text {inside }} d x} \text { and } c_{2}=\frac{\int_{\Omega} I *(1-H(u)) d x}{\int_{\text {inside }} e^{d x}}
$$

Step 3: $\quad u^{n+1}=\left\{\begin{array}{cl}\left(\mathcal{A}_{d} \circ \mathcal{J}_{d} u_{\text {region }}^{n+1}\right)\left(\boldsymbol{x}_{i}\right) & \text { if } g(I)(\boldsymbol{x})>\text { threshold } \\ u_{\text {region }}^{n+1}\left(\boldsymbol{x}_{i}\right) & \text { otherwise. }\end{array}\right.$ 
which is the morphological formulation of the hybrid active contour PDE. The $v$ constant in Step 1 allows us to control the balloon force separate from the smoothing operator. From this, we can directly control the strength of the balloon operator.

\subsection{Implementation Details}

The execution of (20) is trivial, but some specifics regarding the details of the implementation are worth mentioning. First and foremost, regarding the edge detector, our experiments use the builtin Matlab function edge(Img, 'canny',[threshl thresh2],sigma). The input image is a RGB color image, thresh $1=0.2$, thresh $2=0.6$, and sigma $=1$. We maintained these settings for all of our experimental results. The Canny edge detector finds edges by looking for local maxima of the gradient of the image which is calculated using the derivative of a Gaussian filter. Thresh1 and thresh 2 are used to detect strong and weak edges. Weak edges are only included in the edge image if they are connected to strong edges. Sigma is used for the Gaussian kernel.

Also, while it would be simple to extend the morphological operations to grayscale values, the morphological operations used in our implementation are defined as binary operations. The embedded level set is also defined as a binary function. As such, the structuring element for a 3 by 3 window in the mean curvature motion is a square of values of 1 .

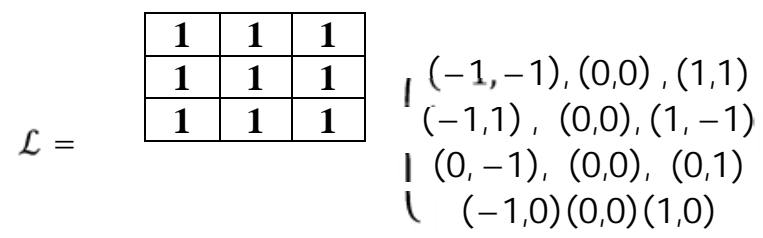

Figure 2: Morphological mean curvature structuring element and associated discrete line segments.

It is not outside the realm of possibility to use line segments of greater length. However, to maintain experimental integrity we use the structuring element in Figure 2 to obtain all of our experimental results.

The region statistics areacquired from the RGB color image rather than its grayscale counterpart. The segmentation algorithm is ran as an unsupervised method in which $u_{0}=g(I), \lambda_{1}=\lambda_{2}=1$, and $v=-1$. The threshold for the balloon force and the mean curvature motion is set to $0.8 *$ $g(I)$. Finally, $|\nabla u|$ is approximated by the magnitude of the gradient, namely $\sqrt{u^{2}+u_{y}{ }^{2}}$ where $u_{x}$ and $u_{y}$ are computed using finite differences.

\subsection{Experimental Results}

Using the natural images available at the Weizmann Segmentation Evaluation Database [30], we performed our algorithm for the images and computed a Sorensen-Dice similarity coefficientfor each image when compared to the provided image ground truth. The average over the segmentation of the data set was 0.9302 which, to the authors' knowledge, is the best Dice score in the literature regarding natural image segmentation. Adjustment of the parameters would naturally have led to better segmentation results for the images which segmented under a 0.9700 similarity coefficient.Table 1 lists statistical information regarding the segmentation of the 204 images in the dataset - 102 images for one object segmentation and 102 images for two object segmentation - while Table 2 gives the segmentation results and Sorensen-Dice coefficients for 
International Journal of Computer Science, Engineering and Applications (IJCSEA) Vol.3, No.4, August 2013

twelve randomly selected segmented images. Figure 3 demonstrates the frequency of the Sorensen-Dice coefficients for the 204 images.

Table 1: Sorensen-Dice Coefficient Results

\begin{tabular}{|c|c|c|}
\hline $\begin{array}{c}\text { Sorensen-Dice } \\
\text { Similarity } \\
\text { Coefficient }\end{array}$ & $\begin{array}{c}\text { 1 Object } \\
\text { Segmentation }\end{array}$ & $\begin{array}{c}\text { 2 Object } \\
\text { Segmentation }\end{array}$ \\
\hline Minimum & 0.6884 & 0.4813 \\
\hline Maximum & 0.9999 & 0.9999 \\
\hline Median & 0.9582 & 0.9692 \\
\hline Mean & 0.9253 & 0.9153 \\
\hline
\end{tabular}

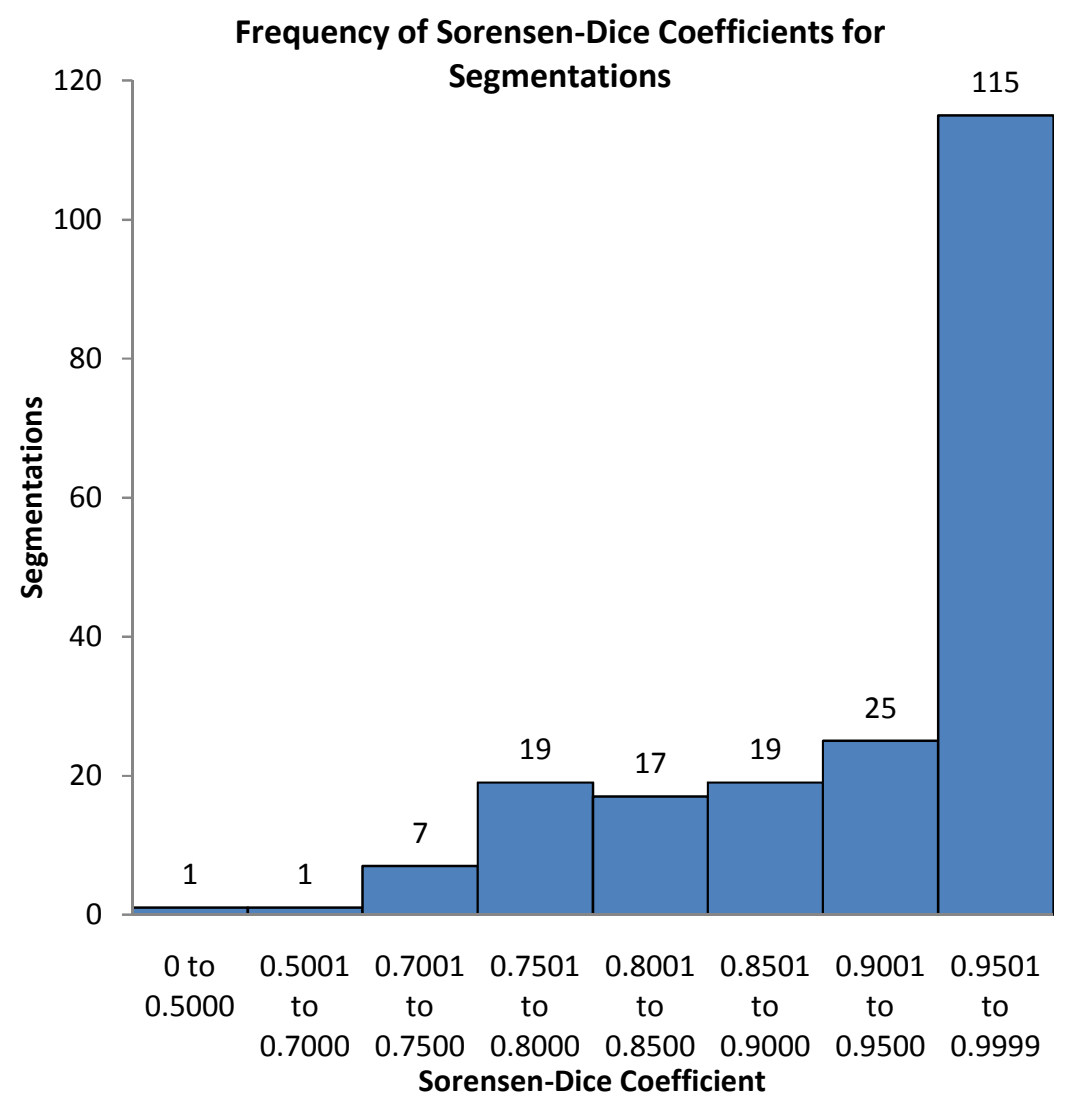

Figure 3: Frequency of Sorensen-Dice Similarity Coefficient for segmentations of 204 images in the dataset when compared to the dataset ground truth. 
International Journal of Computer Science, Engineering and Applications (IJCSEA) Vol.3, No.4, August 2013

Table 2: Segmentation Results of Twelve Randomly Selected Images

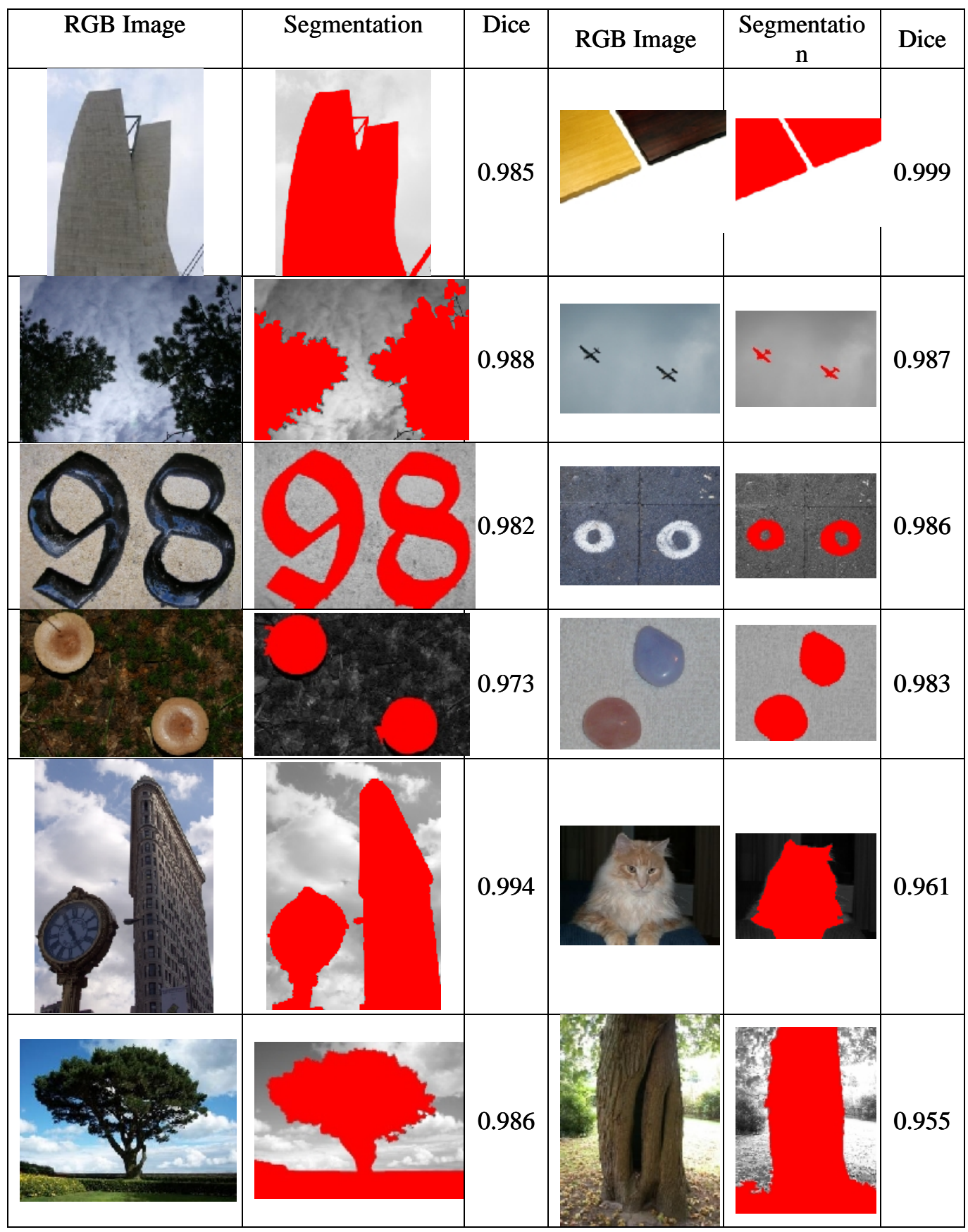

As an example of images in which the proposed method with the stated parameters did not perform well, Table 3 lists the two worst segmentation results from the dataset. While both of the segmentations in Table 3 would have benefitted from parameter tuning, they demonstrate two distinct shortcomings of the method. 
Table 3: Two worst segmentation results from the dataset

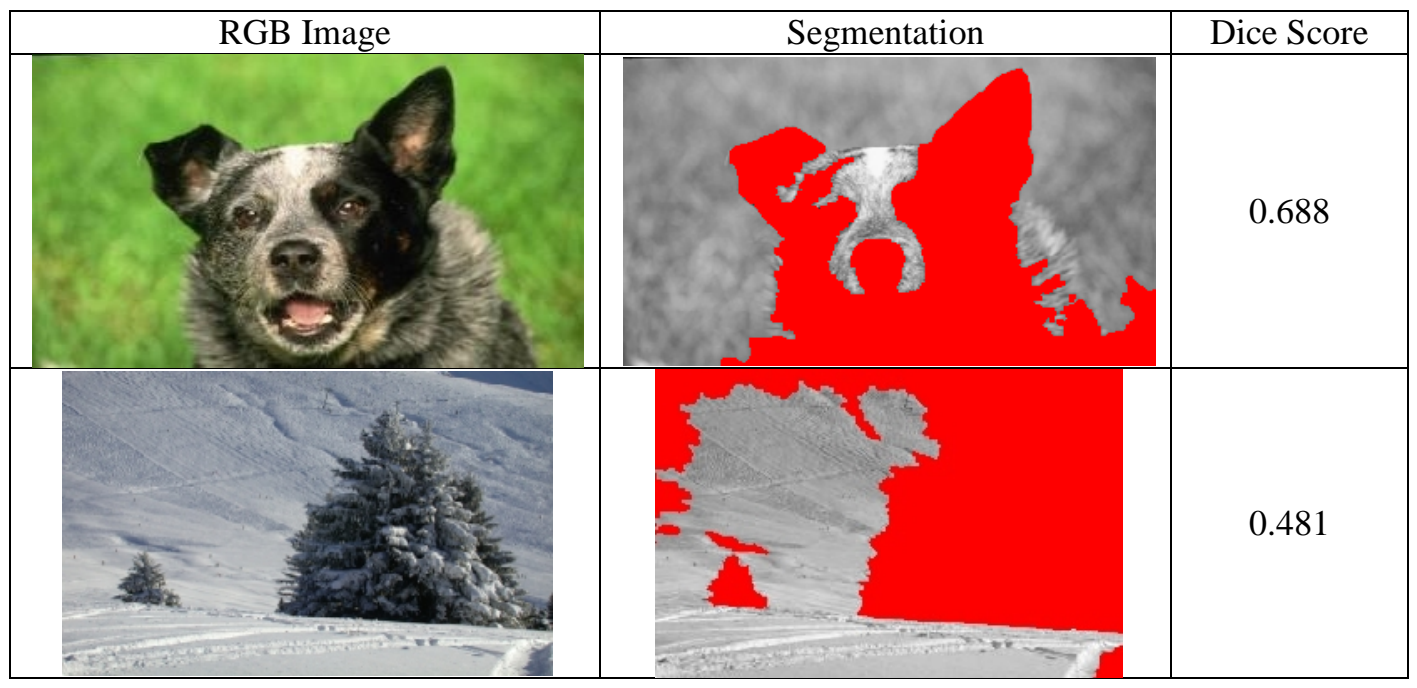

The first image has a clear area of textural homogeneity divided by a sharp intensity difference. As a result the edge term and region term gravitated to the regions with a homogenous intensity. In the second image, there is a clear textural difference between the foreground and background. However, the edges of the region of interest are weak due to the intensity homogeneity across the entire image. As a result, the edge term did not greatly affect the segmentation of the image. To counteract both of these shortcomings, the method could use textural image statistics rather than or in conjunction with intensity statistics. However, incorporating complex region statisticscould negatively impact the overall computation cost of the algorithm.

\section{Conclusions}

This paper introduces a hybrid morphological active contour model. Based on the edge attraction term and mean curvature motion of the Geodesic Active Contour and the region statistics model of the Chan-Vese Active Contour without Edges, the hybrid method accurately and efficiently segmented the natural images in the Weizmann Segmentation Evaluation Dataset. While a few of the dataset images did not segment well with the static parameter settings used for the experiment, the overall Sorenson-Dice similarity coefficient was 0.9302, the highest, to the authors' knowledge, similarity coefficient score for segmentation of natural images in a dataset. Additionally, manual modification of the parameters would yield better segmentation for images that segmented at a lower similarity ratio.

The experiments we have conducted are very promising. The flexibility of the method in the parameter settings and types of statistics that could be incorporated in the region portion of the algorithm could potentially allow for the segmentation of images with sharp intensity gradients across regions of interest or weak edges in areas of intensity homogeneity. In future work, we will experiment with other region statistics for segmentation and extend the method to multispectral images.

\section{ACKNOWLEDGEMENTS}

The authors would like thank the anonymous reviewers for their comments and constructive criticism of this work. Additionally, the authors would like to thank the contributors to the Weizmann Segmentation Evaluation Dataset for the public use of their images and ground-truth segmentations. 


\section{REFERENCES}

[1] Geisler, W. S., \& Diehl, R. L."A Bayesian approach to the evolution of perceptual and cognitive systems."Cognitive Science, 27, 3 (2003):79-402.

[2] Yang, Allen Y., John Wright, Yi Ma, and S. Shankar Sastry. "Unsupervised segmentation of natural images via lossy data compression."Computer Vision and Image Understanding 110, no. 2 (2008): 212-225.

[3] Gao, Shangbing, Jian Yang, and Yunyang Yan. "A novel multiphase active contour model for inhomogeneous image segmentation."Multimedia Tools and Applications (2013): 1-17.

[4] Cremers, Daniel. "Shape Priors for Image Segmentation." In Shape Perception in Human and Computer Vision, pp. 103-117. Springer London, 2013.

[5] Yin, Xue-Min, Hong Yan, Yu-Hua Yao, Jian-Ping Guo, Chong-FaZhong, Zhe Zhang, and Yi Wei. "Multi-region level set image segmentation based on image energy separation model." In Fifth International Conference on Digital Image Processing, pp. 887825-887825. International Society for Optics and Photonics, 2013.

[6] Hedjam, Rachid, and Max Mignotte. "A hierarchical graph-based Markovian clustering approach for the unsupervised segmentation of textured color images." In Image Processing (ICIP), 2009 16th IEEE International Conference on, pp. 1365-1368. IEEE, 2009.

[7] Zhao, Jianhui, Yuanyuan Zhang, Yihua Ding, Chengjiang Long, Zhiyong Yuan, and Dengyi Zhang. "Accelerated Gaussian mixture model and its application on image segmentation." In 2012 International Conference on Graphic and Image Processing, pp. 876831-876831. International Society for Optics and Photonics, 2013.

[8] Arbelaez, Pablo, Michael Maire, CharlessFowlkes, and Jitendra Malik. "Contour detection and hierarchical image segmentation." Pattern Analysis and Machine Intelligence, IEEE Transactions on 33, no. 5 (2011): 898-916.

[9] Mignotte, Max. "A de-texturing and spatially constrained K-means approach for image segmentation."Pattern Recognition Letters 32, no. 2 (2011): 359-367.

[10] Rao, Shankar R., HosseinMobahi, Allen Y. Yang, S. Shankar Sastry, and Yi Ma. "Natural image segmentation with adaptive texture and boundary encoding."In Computer Vision-ACCV 2009, pp. 135-146.Springer Berlin Heidelberg, 2010.

[11] Mignotte, Max. "MDS-based segmentation model for the fusion of contour and texture cues in natural images." Computer Vision and Image Understanding 116, no. 9 (2012): 981-990.

[12] Ciesielski, Krzysztof Chris, Paulo AV Miranda, Alexandre X. Falcão, and Jayaram K. Udupa. "Joint graph cut and relative fuzzy connectedness image segmentation algorithm." Medical Image Analysis (2013).

[13] Tatu, Aditya, and SumukhBansal. "A Novel Active Contour Model for Texture Segmentation." arXiv preprint arXiv:1306.6726 (2013).

[14] Mei, Jiangyuan, Yulin Si, Hamid Reza Karimi, and HuijunGao. "A novel active contour model for unsupervised low-key image segmentation."Central European Journal of Engineering (2013): 1-9.

[15] Kim, Wonjun, and Changick Kim. "Active Contours Driven by the Salient Edge Energy Model." (2013): 1-1.

[16] Li, Chunming, Chiu-Yen Kao, John C. Gore, and Zhaohua Ding. "Minimization of region-scalable fitting energy for image segmentation."Image Processing, IEEE Transactions on 17, no. 10 (2008): 1940-1949.

[17] Rousson, Mikaël, Thomas Brox, and RachidDeriche. "Active unsupervised texture segmentation on a diffusion based feature space." In Computer vision and pattern recognition, 2003. Proceedings.2003 IEEE computer society conference on, vol. 2, pp. II-699.IEEE, 2003.

[18] Cremers, Daniel, Mikael Rousson, and RachidDeriche. "A review of statistical approaches to level set segmentation: integrating color, texture, motion and shape." International journal of computer vision 72, no. 2 (2007): 195-215.

[19] Lankton, Shawn, Delphine Nain, Anthony Yezzi, and Allen Tannenbaum. "Hybrid geodesic regionbased curve evolutions for image segmentation."In Medical Imaging, pp. 65104U65104U.International Society for Optics and Photonics, 2007.

[20] Kimmel, Ron. "Fast edge integration." In Geometric Level Set Methods in Imaging, Vision, and Graphics, pp. 59-77. Springer New York, 2003. 
[21] Kamalakannan, S., S. Antani, R. Long, and G. Thoma. "Customized hybrid level sets for automatic lung segmentation in chest x-ray images." In SPIE Medical Imaging, pp. 866939-866939.International Society for Optics and Photonics, 2013.

[22] Ma, Liyan, and Jian Yu. "An unconstrained hybrid active contour model for image segmentation." In Signal Processing (ICSP), 2010 IEEE 10th International Conference on, pp. 1098-1101. IEEE, 2010.

[23] Ali, Sahirzeeshan, and AnantMadabhushi. "Segmenting multiple overlapping objects via a hybrid active contour model incorporating shape priors: applications to digital pathology."In SPIE Medical Imaging, pp. 79622W-79622W.International Society for Optics and Photonics, 2011.

[24] Welk, Martin, Michael Breuß, and Oliver Vogel. "Morphological amoebas are self-snakes." Journal of Mathematical Imaging and Vision 39.2 (2011): 87-99.

[25] Catté, Francine, Françoise Dibos, and Georges Koepfler. "A morphological scheme for mean curvature motion and applications to anisotropic diffusion and motion of level sets."SIAM Journal on Numerical Analysis 32.6 (1995): 1895-1909.

[26] Álvarez, Luis, Luis Baumela, Pedro Henríquez, and Pablo Márquez-Neila. "Morphological snakes." In Computer Vision and Pattern Recognition (CVPR), 2010 IEEE Conference on, pp. 2197-2202. IEEE, 2010.

[27] Marquez-Neila, Pablo, Baumela, Luis, Alvarez, Luis. "A Morphological Approach to Curvaturebased Evolution of Curves and Surfaces." IEEE Transactions on Pattern Analysis and Machine Intelligence, http://doi.ieeecomputersociety.org/10.1109/TPAMI.2013.106, June 2013.

[28] Kimmel, Ron. Numerical geometry of images: Theory, algorithms, and applications. Springer, 2004.

[29] T. Chan and L. Vese, "Active contours without edges," IEEE Transactions on Image Processing, 10(2):266-277, 2001.

[30] Alpert, Sharon, MeiravGalun, Ronen Basri, and Achi Brandt. "Image segmentation by probabilistic bottom-up aggregation and cue integration."In Computer Vision and Pattern Recognition, 2007.CVPR'07. IEEE Conference on, pp. 1-8. IEEE, 2007.

\section{AUTHORS}

Currently a graduate student in Computational Science at University of Arkansas at Little Rock, Victoria L. Fox is also a mathematics instructor for the University of Arkansas at Monticello. Her professional interests include morphological image processing, segmentation of natural images, and the incorporation of fuzzy logic in multispectral image segmentation.

MariofonnaMilanova is a Professor of Computer Science Department at the University of Arkansas at Little Rock since 2001. She received her M. Sc. degree in Expert Systems and AI in 1991 and her Ph.D. degree in Computer Science in 1995 from the Technical University, Sofia, Bulgaria. Dr.Milanova did her post-doctoral research in visual perception at the University of Paderborn, Germany. She has extensive academic experience at various academic and research organizations in different countries. Milanova serves as a book editor of two books and associate editor of

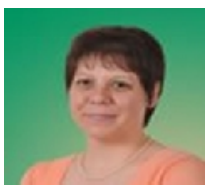
several international journals. Her main research interests are in the areas of artificial intelligence, biomedical signal processing and computational neuroscience, computer vision and communications, machine learning, and privacy and security based on biometric research. She has published and coauthored more than 70 publications, over 43 journal papers, 7 book chapters, numerous conference papers and 2 patents.

Salim Al-Ali is a Ph.D. graduate student in the integrated computing of computer science department at University of Arkansas at Little Rock (UALR). He received a master degree from computer science department, Baghdad University, Iraq on 1995. He is working as a teacher in Dohuk Technical Institute at Dohuk Polytechnic University. His research interest field is computer vision in general, human action recognition, image and video understanding.

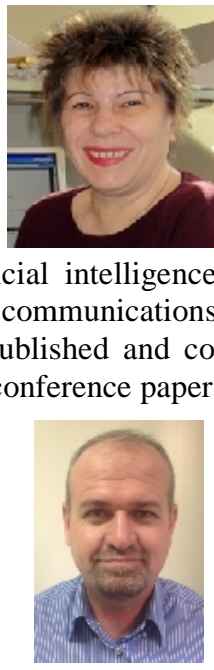

